\title{
Exact ground state in an orbitally degenerate Hubbard model
}

\author{
Shun-Qing Shen* \\ Department of Physics, Tokyo Institute of Technology, Oh-okayama, Meguro-ku, Tokyo 152, Japan
}

(Received 22 September 1997; revised manuscript received 12 November 1997)

\begin{abstract}
We present an exact solution on the ground state of a one-dimensional orbitally degenerate Hubbard model in the case of strong coupling, and the phase diagram in terms of electron filling and relevant parameters. The ground state is nondegenerate apart for spin SU(2) symmetry and a fully saturated and metallic ferromagnet except the case of the quarter filling, at which a ferromagnetic Mott insulator transition occurs. The phase diagram shows coexistence of ferromagnetism and spin-triplet pairing superconductivity when the Hund coupling is sufficiently larger. A mechanism for spin-triplet pairing superconductivity and its possible relevance to the quasi-one-dimensional organic superconductor are proposed. [S0163-1829(98)00311-7]
\end{abstract}

\section{INTRODUCTION}

Orbital degrees of freedom enrich the phase diagram of a strongly correlated electron system. Interplay of orbital and spin degrees of freedom produces a series of novel physical phenomena, such as metal-insulator transition, spin and orbital ordered states, and transport property anomaly. However, the complexity of strong correlation in these systems prevents us from a complete and deep understanding of physical properties by means of conventional approaches. As a theoretically simplified model, the orbital degenerate Hubbard model is attracting recent attention. Various approximations and computation methods, such as the perturbative projection technique, slave-boson, and dynamical mean field theory, and the quantum Monte Carlo method, are attempted to investigate the system. ${ }^{1-11} \mathrm{We}$ expect to extract some rigorous results from the theoretical model, which are believed to be helpful for us to test the validity of approximate and numerical results.

Physically, metallic ferromagnetism is one of the most important, but fully mysterious phenomena in condensed matter physics. Recently, the understanding of ferromagnetism in strongly correlated electron system has made a lot of progresses. ${ }^{12-17}$ However all rigorous results are restricted to the insulating case. Relation of superconductivity and ferromagnetism is a long standing problem. ${ }^{18}$ The coexistence of superconductivity and ferromagnetism is prospective for both theoretical curiosity and application. It would break through the limit of the conventional Bardeen-CooperSchrieffer (BCS) theory of superconductivity, and will provide a promising routine for looking for a superconductor with a high critical magnetic field.

An explicit form of the Hamiltonian for an orbitally degenerate Hubbard model is written as

$$
H=H_{t}+H_{U}+H_{J},
$$

where

$$
H_{t}=\sum_{i, j, \gamma, \gamma^{\prime}, \sigma} t_{i j}^{\gamma \gamma^{\prime}} c_{i, \gamma, \sigma}^{\dagger} c_{j, \gamma, \sigma}
$$

where $c_{i, \gamma, \sigma}^{\dagger}$ and $c_{i, \gamma, \sigma}$ are creation and annihilation operators for an electron with spin $\sigma(=\uparrow, \downarrow)(\bar{\sigma}$ is complimentary of $\sigma)$ at site $i$ on orbital $\gamma(=1,2)$, respectively. This model consists of $N_{\wedge}$ sites and 2 orbitals on each site. In this paper we consider a one-dimensional chain and take (i) the hopping matrix $t_{i j}^{\gamma \gamma^{\prime}}=-t \delta_{\gamma, \gamma^{\prime}} \delta_{|i-j|, 1}$, (ii) the on-site interaction of the same orbital $U_{\gamma \gamma}=U$, (iii) the on-site interaction of the different orbital $U_{\gamma \gamma^{\prime}}=U^{\prime}\left(\gamma \neq \gamma^{\prime}\right)$, and (iv) the Hund coupling between electrons of different orbitals $J_{\gamma \gamma^{\prime}}=J(\gamma$ $\left.\neq \gamma^{\prime}\right)$. Define the spin operator of electron $\mathbf{S}_{i, \gamma}$ $=\Sigma_{\sigma, \sigma^{\prime}} c_{i, \gamma, \sigma}^{\dagger}(\boldsymbol{\sigma})_{\sigma, \sigma^{\prime}} c_{i, \gamma, \sigma^{\prime}}$ (where $\boldsymbol{\sigma}$ are Pauli matrices). In this model the total spin operator $\mathbf{S}_{\text {tot }}=\Sigma_{i, \gamma} \mathbf{S}_{i, \gamma}$ commutes with the Hamiltonian $\left[\mathbf{S}_{\mathrm{tot}}, H\right]=0$ and is a good quantum number. The maximum of total spin is $N_{e} / 2\left(N_{e}\right.$ is the number of electrons and $\left.N_{e}<2 N_{\wedge}\right)$. We call a state with the maximum of total spin a fully saturated ferromagnet. Due to spin $\mathrm{SU}(2)$ symmetry, the state is always $\left(N_{e}+1\right)$-fold degenerate. Another hidden symmetry is of orbital degree of freedom: $\mathbf{T}_{i, \sigma}=\Sigma_{\gamma, \gamma^{\prime}} c_{i, \gamma, \sigma}^{\dagger}(\boldsymbol{\sigma})_{\gamma, \gamma^{\prime}} c_{i, \gamma, \sigma^{\prime}}$. These operators also obey SU(2) algebra just as spin operators do. The total orbital spin commutes with the Hamiltonian when there is no hopping between different orbitals in $H_{t}$.

In this paper we present a set of exact solutions on the ground state of a one-dimensional orbital degenerate Hubbard model in the case of strong coupling by means of the variational principle and the Bethe ansatz. We obtain a phase diagram for this system. The ground state is a metallic ferromagnet, except for the quarter filled case, in which a ferromagnetic Mott-insulator transition occurs. In the case of the strong Hund coupling between electrons on different orbitals, we discuss the coexistence of ferromagnetism and superconductivity, and its possible relevance with recent experimental observation of superconductivity in a quasi-onedimensional sample in the presence of a high magnetic field. 


\section{THE GROUND STATE SOLUTION}

\section{A. Exact solution of the ground state}

Even for the one-dimensional case, it is still very difficult to solve the model exactly or to extract rigorous results from the model. Some numerical and analytical calculations were done. In the present paper, we limit our discussion to the case of strong coupling, i.e., $U \rightarrow+\infty$ so that the double occupancy of electrons on the same site of the same orbital is excluded. An equivalent Hamiltonian of Eq. (1) in the large $U$ limit is reduced to

$$
H=H_{1}+\sum_{i} H_{2 i}
$$

where

$$
\begin{gathered}
H_{1}=t \sum_{i, \delta, \gamma, \sigma}\left(1-n_{i, \gamma, \bar{\sigma}}\right) c_{i, \gamma, \sigma}^{\dagger} c_{i+\delta, \gamma, \sigma}\left(1-n_{i+\delta, \gamma, \bar{\sigma}}\right) \\
+U_{\text {eff }} \sum_{i, \gamma \neq \gamma^{\prime}, \sigma, \sigma^{\prime}} P_{D} n_{i, \gamma, \sigma} n_{i, \gamma^{\prime}, \sigma^{\prime}} P_{D}, \\
H_{2 i}=J \sum_{\gamma \neq \gamma^{\prime}, \sigma} P_{D}\left(c_{i, \gamma, \sigma}^{\dagger} c_{i, \gamma, \sigma} c_{i, \gamma^{\prime}, \bar{\sigma}}^{\dagger} c_{j, \gamma^{\prime}, \bar{\sigma}}\right. \\
\left.-c_{i, \gamma, \sigma}^{\dagger} c_{i, \gamma, \bar{\sigma}} c_{i, \gamma^{\prime}, \bar{\sigma}}^{\dagger} c_{j, \gamma^{\prime}, \sigma}\right) P_{D},
\end{gathered}
$$

$P_{D}=\Pi_{i, \gamma}\left(1-n_{i, \gamma, \uparrow} n_{i, \gamma, \downarrow}\right)$ and $U_{\mathrm{eff}}=U^{\prime}-J$.

To establish a rigorous result, let us consider $H_{1}$ and $H_{2}$ $=\Sigma_{i} H_{2 i}$, respectively. In the variational principle if we can find a state which is simultaneously the lowest energy state of both $H_{1}$ and $H_{2}$, then it must be the ground state of $H$.

We first introduce a set of basis for this system. Assume $N_{1}$ electrons on $\gamma=1$ orbital, and $N_{2}$ electrons on $\gamma=2$ orbital. $N=N_{1}+N_{2}$. The $N_{1}$ electrons on $\gamma=1$ orbital with spin $\sigma_{1}, \sigma_{2}, \ldots, \sigma_{N_{1}}$ are located at $x_{1}<x_{2}<\ldots<x_{N_{1}}$. The $N_{2}$ electrons on $\gamma=2$ orbital with spin $\sigma_{N_{1}+1}, \sigma_{N_{1}+2}, \ldots, \sigma_{N}$ are located at $x_{N_{1}+1}<x_{N_{1}+2}<\cdots$ $<x_{N}$. A state on this basis is expanded as

$$
\begin{aligned}
|\Psi\rangle= & \sum_{\left\{x_{i}\right\},\left\{\gamma_{i}\right\},\left\{\sigma_{i}\right\}} \\
& \times f\left(x_{1}, \ldots, x_{N} ; \gamma_{1}, \ldots, \gamma_{N} ; \sigma_{1}, \ldots, \sigma_{N}\right) \\
& \times c_{x_{1}, \gamma_{1}, \sigma_{1}}^{\dagger} c_{x_{2}, \gamma_{2}, \sigma_{2}}^{\dagger} \cdots c_{x_{N}, \gamma_{N}, \sigma_{N}}^{\dagger}|0\rangle,
\end{aligned}
$$

where $|0\rangle$ is the vacuum state. The Schrödinger equation for $f$ in $H_{1}(\operatorname{not} H)$ is

$$
\begin{aligned}
& -t \sum_{i, \delta, \gamma} f\left(x_{1}, \ldots, x_{i}+\delta, \ldots, x_{N} ; \gamma_{1}, \ldots, \gamma_{N} ; \sigma_{1}, \ldots, \sigma_{N}\right) \\
& \quad+U_{\text {eff }} \sum_{i<j} \delta_{x_{i}, x_{j}}\left(1-\delta_{\gamma_{i}, \gamma_{j}}\right) \\
& \quad \times f\left(x_{1}, \ldots, x_{N} ; \gamma_{1}, \ldots, \gamma_{N} ; \sigma_{1}, \ldots, \sigma_{N}\right) \\
& =E f\left(x_{1}, \ldots, x_{N} ; \gamma_{1}, \ldots, \gamma_{N} ; \sigma_{1}, \ldots, \sigma_{N}\right)
\end{aligned}
$$

with the boundary conditions

$$
\left.f\left(x_{1}, \ldots, x_{N} ; \gamma_{1}, \ldots, \gamma_{N} ; \sigma_{1}, \ldots, \sigma_{N}\right)\right|_{x_{i}=x_{j}, \gamma_{i}=\gamma_{j}}=0,
$$

for all $i<j$. The boundary conditions do not depend on spin indices. The conditions for $\sigma_{i}=\sigma_{j}$ comes from the Pauli exclusion principle, and the conditions for $\sigma_{i}=-\sigma_{j}$ from the strong coupling limit on the same orbital. An observation in Eq. (6) is that the eigenvalues $E$ are independent of spin distribution: the wave functions with different spin distributions satisfy the same set of equations. In other words, the spin degree of freedom is decoupled completely with the charge and orbital degrees of freedom in the wave function, which is similar to the one-band Hubbard model in the large $U$ limit. ${ }^{19}$ The wave function can be written in the form

$$
\begin{aligned}
& f\left(x_{1}, \ldots, x_{N} ; \gamma_{1}, \ldots, \gamma_{N} ; \sigma_{1}, \ldots, \sigma_{N}\right) \\
& \quad=g\left(x_{1}, \ldots, x_{N} ; \gamma_{1}, \ldots, \gamma_{N}\right) \phi\left(\sigma_{1}, \ldots, \sigma_{N}\right) .
\end{aligned}
$$

$\phi$ is an arbitrary function of spin distribution. Substituting Eq. (7) into Eq. (6) we obtain

$$
\begin{aligned}
& -t \sum_{i, \delta, \gamma} g\left(x_{1}, \ldots, x_{i}+\delta, \ldots, x_{N} ; \gamma_{1}, \ldots, \gamma_{N}\right)+\left(U^{\prime}-J\right) \\
& \quad \times \sum_{i<j} \delta_{x_{i}, x_{j}}\left(1-\delta_{\gamma_{i}, \gamma_{j}}\right) g\left(x_{1}, \ldots, x_{N} ; \gamma_{1}, \ldots, \gamma_{N}\right) \\
& =E g\left(x_{1}, \ldots, x_{N} ; \gamma_{1}, \ldots, \gamma_{N}\right)
\end{aligned}
$$

with

$$
\left.g\left(x_{1}, \ldots, x_{N} ; \gamma_{1}, \ldots, \gamma_{N}\right)\right|_{x_{i}=x_{j}, \gamma_{i}=\gamma_{j}}=0
$$

for all $i<j$. These equations for $g$ are equivalent to those for a one-band Hubbard model with $U_{\text {eff }}$ if we regard the orbital indices in $g$ as usual spin indices in the one-band Hubbard model. The solution for $g$ is expressed by means of the Bethe ansatz as ${ }^{20}$

$$
g\left(x_{1}, \ldots, x_{N} ; \gamma_{1}, \ldots, \gamma_{N}\right)=\sum_{P}[Q, P] \exp \left[i \sum_{j=1}^{N} k_{P_{j}} x_{Q_{j}}\right] \text {, }
$$

where $P$ and $Q$ are two permutation of $(1,2, \ldots, N)$. The coefficients $[Q, P]$ are not independent of each other:

$$
\begin{gathered}
{[Q, P]=Y_{n m}^{i, i+1}\left[Q, P^{\prime}\right] ;} \\
Y_{n m}^{i, i+1}=\frac{\left(\sin k_{n}-\sin k_{m}\right) P^{i, i+1}-i U_{\mathrm{eff}} / 2}{\left(\sin k_{n}-\sin k_{m}\right)+i U_{\mathrm{eff}} / 2}
\end{gathered}
$$

where $P=\left(P_{1}, \ldots, P_{i}=n, P_{i+1}=m, \ldots, P_{N}\right)$ and $P^{\prime}$ $=\left(P_{1}, \ldots, P_{i}^{\prime}=m, P_{i+1}^{\prime}=n, \ldots, P_{N}\right)$.

Now we come to consider $H_{2 i}$. For each site $i$, there are four configurations of electrons after we exclude the double occupancy of electrons on the same site of the same orbital: empty, single occupancy and double occupancy of electrons on the same site of different orbitals. The double occupancy is characterized by spin singlet and spin triplet. The interaction energy of $H_{2 i}$ on a single site is $J$ for double occupancy of spin singlet, and zero for other three configurations. Hence the lowest energy of $\mathrm{H}_{2}$ for electrons $N \leqslant 2 N_{\wedge}$ is always zero, and the state does not consist of on-site spin singlet. Suppose $|\Psi(g, \phi)\rangle$ one of the lowest energy states of $H_{1}$ 
with an arbitrary spin distribution $\phi . g$ is the lowest energy wave function in Eq. (9). The average energy of $H_{2}$ on this state is expressed as

$$
\left\langle\Psi(g, \phi)\left|H_{2}\right| \Psi(g, \phi)\right\rangle=\sum_{i<j} \delta_{x_{i}, x_{j}} \rho\left(x_{i}, x_{j}\right) s\left(x_{i}, x_{j}\right),
$$

where

$$
\begin{aligned}
\rho\left(x_{i}, x_{j}\right)= & \sum_{x_{k}, k \neq i, j} g^{*}\left(x_{1}, \ldots, x_{i}, \ldots, x_{j}, \ldots, x_{N}\right) \\
& \times g\left(x_{1}, \ldots, x_{i}, \ldots, x_{j}, \ldots, x_{N}\right) \geqslant 0, \\
& s\left(x_{i}, x_{j}\right)=\left\langle\phi\left|\left(1-P_{i j}^{\sigma}\right)\right| \phi\right\rangle .
\end{aligned}
$$

$P_{i j}^{\sigma}$ is the permutation operator of spins at $i$ and $j$. The eigenvalue is +1 when spins on $i$ and $j$ form triplet, and is -1 when spins form singlet. Physically, $s\left(x_{i}, x_{j}\right)$ is the possibility of forming a spin singlet at site $x_{i}=x_{j}(i \neq j)$ and $\rho\left(x_{i}, x_{j}\right)$ is the possibility of double occupancy of electron at site $x_{i}=x_{j}$. For finite $U_{\text {eff }}=U^{\prime}-J, \rho\left(x_{i}, x_{j}\right)$ is not zero in the lowest energy state. $\rho\left(x_{i}, x_{j}\right)=n / 4$ when $U_{\text {eff }}=0$. $\rho\left(x_{i}, x_{j}\right)=n / 2$ as $U_{\text {eff }}$ approaches $-\infty$, and 0 as $U_{\text {eff }}$ approaches $+\infty$. To obtain the lowest energy of $\mathrm{H}_{2}$, all $s\left(x_{i}, x_{j}\right)$ have to be zero. This condition can be satisfied by a fully saturated ferromagnetic state. Hence we have to choose $\phi$ the state with maximum of the total spin so that $|\Psi(g, \phi)\rangle$ is the lowest energy state of $\mathrm{H}_{2}$.

Since the the ferromagnetic state $|\Psi(g, \phi)\rangle$ is the lowest energy state of both $\mathrm{H}_{1}$ and $\mathrm{H}_{2}$ in Eq. (2) simultaneously, according to the variational principle, we draw a conclusion that the state $|\Psi(g, \phi)\rangle$ is the ground state of $H$ in Eq. (1) in the case of large $U$ limit. (The conclusion of ferromagnetism in the ground state is also true when the system contains more than two orbitals.)

\section{B. Nondegeneracy of the ground state}

The ground state is nondegenerate when $J>0, U^{\prime}$ is finite and $N_{e}<2 N_{\wedge}$. This is proved by using Perron-Frobenius theorem. ${ }^{21}$ The theorem states that for a real, symmetric, and square matrix $M=\left\{m_{i j}\right\}$ if (i) all its off-diagonal matrix elements are nonpositive $\left[m_{i j} \leqslant 0\right.$ for any $\left.(i \neq j)\right]$, and (ii) for any $i, j$ we can always find an integer $n$ such that $\left(M^{n}\right)_{i j}$ $\neq 0$, its eigenvector with lowest value is unique and all elements in the vector are positive. In my problem here the Hamiltonian can be expressed in the form of sqaure matrix on the basis I choose. The Hamiltonian matrix satisfies the conditions of the Perron-Frobenius theorem.

(i) Nonpositive off-diagonal matrix elements: Express $H$ in a square matrix on the basis we choose. The nonzero diagonal matrix elements are $U^{\prime}$ and $J$ due to the on-site density-density interaction and the Hund coupling between electrons in different orbitals, respectively. The nonzero offdiagonal elements are $-t$ due to the hopping terms, and $-J$ due the Hund coupling. As both $-t$ and $-J$ are negative, all nonzero off-diagonal matrix elements of $H$ are nonpositive.

(ii) All basis are connected through $H$ : (a) the hopping terms connect all lattice sites, (b) finite $U^{\prime}$ allows the on-site double occupancy of electrons on different orbitals, and (c) the double occupancy and the Hund coupling allows permutation of spin indices of electrons on the same site. Combination of (a), (b), and (c) allows permutation of spin indices of electrons on the same orbital when the system is not half filled, i.e., $N_{e}=2 N_{\wedge}$. Successive applications of (a), (b), and (c) prove that all basis are connected.

From (i) and (ii), we come to conclude that under the conditions the ground state of $H$ is nondegenerate. All coefficients are in the ground state [see Eq. (5)] :

$$
f\left(x_{1}, \ldots, x_{N} ; \gamma_{1}, \ldots, \gamma_{N} ; \sigma_{1}, \ldots, \sigma_{N}\right)>0 .
$$

The property provides us with an alternative way to show ferromagnetism in the ground state. We construct a state with all constant coefficients $c,|\phi\rangle$. This state possesses the maximal total spin $N_{e} / 2$ if $N_{e}<2 N_{\wedge} .{ }^{22}$ Since overlap between the ground state and the state $|\phi\rangle$ is always nonzero,

$$
\langle\Psi \mid \phi\rangle=c \sum_{\left\{x_{i}\right\},\left\{\gamma_{i}\right\},\left\{\sigma_{i}\right\}} f \neq 0,
$$

and both states are eigenstates of the total spin, they must have the same total spin. ${ }^{23}$

\section{THE PHASE DIAGRAM OF THE GROUND STATE}

Except for the fully saturated ferromagnetism in the ground state $|\Psi\rangle$ of $H$, other physical properties related to the charge and orbital degrees of freedom are determined by solution $g$, which depends on the effective interaction $\widetilde{U}_{\text {eff }}$ and the electron filling. Formally $g$ can be determined by Eq. (9). This solution has been discussed extensively. ${ }^{24}$ For $U_{\text {eff }}>0$, i.e., $U^{\prime}>J, g$ is a solution for a positive $U$ Hubbard model, and when $U_{\text {eff }}<0, g$ is a solution for a negative $U$ Hubbard model. When $U^{\prime}=0, g$ is a solution of free fermion gas with orbital degeneracy.

Using the technique of bosonization, ${ }^{25}$ the low-energy charge and orbital density excitations are described by the effective Hamiltonian

$$
H \approx H_{c}+H_{o},
$$

where

$$
\begin{aligned}
& H_{c}=\frac{1}{2} \int d x\left[\Pi_{c}^{2}+\left(\partial \phi_{c}\right)^{2} A_{c}^{2}\right]-\frac{\delta_{n, 1} U_{\text {eff }}}{2 \pi^{2} \alpha^{2}} \cos \left(\sqrt{8 \pi} \phi_{c}\right) ; \\
& H_{o}=\frac{1}{2} \int d x\left[\Pi_{o}^{2}+\left(\partial \phi_{o}\right)^{2} A_{o}^{2}\right]+\frac{U_{\text {eff }}}{2 \pi^{2} \alpha^{2}} \cos \left(\sqrt{8 \pi} \phi_{o}\right) .
\end{aligned}
$$

$\phi_{c}$ and $\phi_{o}$ are the charge and orbital fields, respectively. $A_{c}^{2}=1+U_{\text {eff }} / \pi$ and $A_{o}^{2}=1-U_{\text {eff }} / \pi$. The charge excitation is massless except for the case of quarter filling, i.e., $n$ $=N / N_{\wedge}=1$. This indicates that the low energy charge excitation has no gap, and the system will be metallic.

\section{A. Ferromagnetic Mott-insulator transition: Quarter filling}

In the case of quarter filling, the charge excitation is massive when $U_{\text {eff }}>0$, but massless when $U_{\text {eff }}<0$. The energy gap for $U_{\text {eff }}>0$ is expressed exactly as 


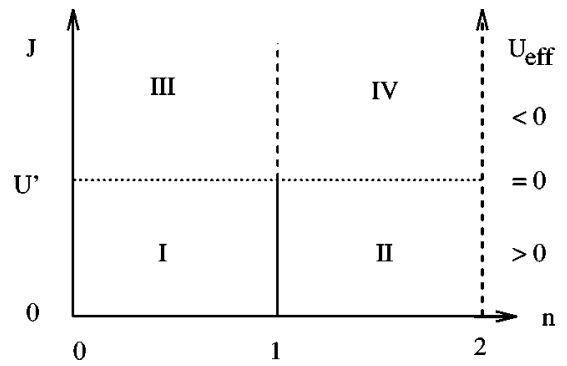

FIG. 1. The phase diagram of the one-dimensional model for the charge and orbital isospin degrees of freedom in a fully saturated ferromagnetic state. The solid line is of $n=1$ and $J<U^{\prime}$, and represents an insulating state. Phases I and II are an solution for a positive $U$ Hubbard model, and phases III and IV are for an attractive $U$ Hubbard model. There is a quantum phase transition near the dashed line at $J=U^{\prime}$. Phases I and II are related to each other through the particle-hole transformation, as are phases III and IV.

$$
\Delta=4 t \int_{0}^{\infty}[\omega \lambda-\tanh (\omega \lambda)] \frac{J_{1}(\omega)}{\omega} d \omega>0,
$$

where $\lambda=U_{\text {eff }} / 4 t$ and $J_{1}(\omega)$ is the first Bessel function according to Lieb-Wu solution. ${ }^{20}$ The gap disappears when $U_{\text {eff }}<0$. Hence there is a ferromagnetic Mott-insulator transition when the system is quarter filled. The low energy equation for the orbital excitation is very similar to that for the charge excitation at quarter filling, but the signs of interaction are opposite. When $U_{\text {eff }}>0$, the orbital excitation is massless, but when $U_{\text {eff }}<0$, the orbital excitation is massive, i.e., the low-energy orbital excitation has a gap. In fact the charge excitation at quarter filling and orbital excitation has a dual relation under a partial particle-hole transformation, which will map a positive $U_{\text {eff }}$ case to a negative case meanwhile the the charge excitation is mapped onto the orbital excitation and vice versa. A schematic phase diagram is shown in Fig. 1.

\section{B. Coexistence of ferromagnetism and superconductivity}

A more interesting phenomenon in the ground state occurs in the case of $U_{\text {eff }}<0$. Apart from spin $\mathrm{SU}(2)$ symmetry, there is an additional SU(2) symmetry for orbital degree of freedom. We have shown that the orbital excitation has a gap when $U_{\text {eff }}<0$. A physical argument is that when $U_{\text {eff }}$ $<0$ electrons with opposite orbital spins and parallel spins tend to form spin triplet and orbital singlet. If we want to make an orbital excitation, we must break a pair, which will cost a finite energy. Following Bogoliubov and Korepin, ${ }^{26}$ we find that the correlation functions of electron pairs with spin triplet and orbital spin singlet decays in a power law

$$
\left\langle\Psi\left|c_{i, 1, \sigma}^{\dagger} c_{i, 2, \sigma}^{\dagger} c_{i+n, 2, \sigma} c_{i+n, 1, \sigma}\right| \Psi\right\rangle \rightarrow \frac{1}{n^{\alpha}}, \quad \text { as } \quad n \rightarrow \infty,
$$

with where $v_{F}$ is the Fermi velocity and $\kappa$ is the compressibility, and $\xi>0$. Meanwhile the correlation function of single electrons decays exponentially:

$$
\left\langle\Psi\left|c_{i, \gamma, \sigma^{\prime}}^{\dagger} c_{i+n, \gamma^{\prime}, \sigma^{\prime}}\right| \Psi\right\rangle \rightarrow \delta_{\gamma, \gamma^{\prime}} \delta_{\sigma, \sigma^{\prime}} e^{-n / \xi}, \quad \text { as } n \rightarrow \infty .
$$

This indicates that the ground state has strong instability of superconductivity. In a realistic sample the quasi-one structure or small hopping between one-dimensional chains could suppress the quantum fluctuation and enhance the tendency to form superconductivity. Hence the gound state of $U_{\text {eff }}$ $<0$ is a coexistence of ferromagnetism and superconductivity.

\section{DISCUSSIONS}

The coexistence of ferromagnetism and superconductivity is a phenomenon in condensed matter. There have been a lot of theoretical investigations of spin-triplet pairing superconductivity. ${ }^{27-30}$ (Liquid ${ }^{3} \mathrm{He}$ is a spin-triplet superfluid. ${ }^{31}$ ) Shelton and Tsvelik ${ }^{11}$ presented a onedimensional example that superconductivity of spin triplet pairs occurs in the case of finite $U$ by using the bosonization technique. As far as I know there is no discussion on coexistence of ferromagnetism and superconductivity. At present there is some indirect experimental evidence to support the coexistence of ferromagnetism and superconductivity. Recent experiment on a quasi-one dimensional organic superconductor (TMTSF) ${ }_{2} \mathrm{PF}_{6}$ by Lee et al. ${ }^{32}$ shows that superconductivity survives in a high magnetic field. In a spin singlet pairing superconductor, electron pairing takes place between electrons with opposite spins, but electron spins tend to align parallel in a magnetic field. The limit of the paramagnetic limiting field for breaking an isotropic pairing is given by $H_{p}=1.84 T_{c}$ where $T_{c}$ is the critical temperature. ${ }^{33}$ Compared with spin-singlet pairing, the spintriplet superconductor is not limited by this magnetic field although $H_{p}$ is usually much larger than the critical field $H_{c} \cdot{ }^{29}$ (TMTSF) ${ }_{2} \mathrm{PF}_{6}$ is a type-II superconductor. Lee et al. measured the critical magnetic field $H_{c 2}$ along the $c$ direction and found that $H_{c 2}$ is much larger than $H_{p}$, which suggests that full polarization of electron spins takes place in the sample.

Here we make a theoretical speculation, although more evidence is needed to relate our work with the superconductivity in (TMTSF) ${ }_{2} \mathrm{PF}_{6}$. Our soluble model provides a possible mechanism of superconductivity with fully polarized spins. When we consider the Hund coupling between electrons on different chains [in (TMTSF) ${ }_{2} \mathrm{PF}_{6}$ it should be on chains, not orbitals], electrons on different chains tend to form a spin-triplet pair, which has a lower energy than spin singlet pair. When the Hund coupling is stronger that the on-site interaction $U^{\prime}$, the electrons with parallel spins on different chains will "feel" an attractive interaction, which will drive the electrons to form spin-triplet, and orbital spinsinglet pairs, further to form superconductivity. In presence of an external magnetic field the spin fluctuation is suppressed and it is easier to form a ferromagnetic state, in which the attractive interaction is enhanced. In other word, the external magnetic field will enhance the superconductivity in our model, not suppress superconductivity as in a conventional BCS superconductor. 
In summary, we obtain the exact solution of the nondegenerate ground state of an orbital degenerate Hubbard model in the strong coupling limit [apart for $(2 S+1)$-fold $\mathrm{SU}(2)$ degeneracy]. We showed that the state is fully saturated ferromagnetic. At quarter filling, a ferromagnetic Mott insulator occurs, which depends on the difference between the on-site interaction and the Hund coupling. Except for quarter filling, the charge excitation is gapless and the state is metallic. When the Hund coupling becomes strong, the charge and orbital part of the wave function is determined by an attractive one-band Hubbard model. It indicates that Hund strong coupling will lead to proof that ferromagnetism and superconductivity coexit. In this case we expect that superconductivity survives in the presence of a relatively higher magnetic field.

\section{ACKNOWLEDGMENTS}

The author thanks Professor H. Shiba for his encouragement and discussions, and Professor H. Tasaki for his discussion. This work was supported by the Japan Society for the Promotion of Science.
*Present address: Department of Physics, The University of Hong Kong, Pokfulam Road, Hong Kong. Electronic address: sshen@hkucc.hku.hk

${ }^{1}$ L. M. Roth, Phys. Rev. 149, 306 (1966); J. Appl. Phys. 38, 1065 (1967)

${ }^{2}$ K. I. Kugel and D. I. Khomskii, Sov. Phys. JETP 37, 725 (1973); Sov. Phys. Usp. 25, 231 (1982).

${ }^{3}$ B. Sutherland, Phys. Rev. B 12, 3795 (1975).

${ }^{4}$ B. H. Brandow, Adv. Phys. 26, 651 (1977).

${ }^{5}$ P. Schlottmann, Phys. Rev. B 43, 3101 (1991).

${ }^{6}$ J. P. Lu, Phys. Rev. B 49, 5687 (1994).

${ }^{7}$ G. Kotliar and H. Kajueter, Phys. Rev. B 54, 14221 (1996).

${ }^{8}$ H. Shiba, R. Shiina, and A. Takahashi, J. Phys. Soc. Jpn. 66, 941 (1997).

${ }^{9}$ Y. Motome and M. Imada, J. Phys. Soc. Jpn. (to be published).

${ }^{10}$ R. Fresard and G. Kotliar, Phys. Rev. B 56, 12909 (1997).

${ }^{11}$ D. G. Shelton and A. M. Tsvelik, Phys. Rev. B 53, 14036 (1996).

${ }^{12}$ E. H. Lieb, Phys. Rev. Lett. 62, 1201 (1989); 62, 1927(E) (1989). The antiferromagnetic long-range correlation in Lieb's ferrimagnet was proved by Shen et al. (Ref. 13).

${ }^{13}$ S. Q. Shen, Z. M. Qiu, and G. S. Tian, Phys. Rev. Lett. 72, 1280 (1994).

${ }^{14}$ A. Mielke, J. Phys. A 24, L73 (1991); 24, 3311 (1991).

${ }^{15}$ H. Tasaki, Phys. Rev. Lett. 69, 1608 (1992); 75, 4678 (1995).

${ }^{16}$ R. Strack and D. Vollhardt, Phys. Rev. Lett. 72, 3425 (1994).

${ }^{17}$ E. Müller-Hartmann, J. Low Temp. Phys. 99, 349 (1995).

${ }^{18}$ P. W. Anderson and B. T. Matthias, Science 144, 373 (1964).

${ }^{19}$ M. Ogata and H. Shiba, Phys. Rev. B 41, 2326 (1990).

${ }^{20}$ E. H. Lieb and F. Y. Wu, Phys. Rev. Lett. 20, 1445 (1968).
${ }^{21}$ Kubo first used the theorem to prove ferromagnetism in a onedimensional double exchange mode, see, K. Kubo, J. Phys. Soc. Jpn. 51, 782 (1982).

${ }^{22}$ In the case of $N_{e}>2 N_{\wedge}$, the ground state is still nondegenerate and pocesses nonzero spin $\left(4 N_{\wedge}-N_{e}\right) / 2$. The ground state wave function can be obtained from the wave function of $N_{e}<2 N_{\wedge}$ by using a particle-hole transformation.

${ }^{23}$ S. Q. Shen, Phys. Rev. B 53, 14252 (1996).

${ }^{24}$ For a review, see N. Andrei, in Low-dimensional Quantum Field Theory for Condensed Matter Physicists, edited by S. Lundquist et al. (World Scientific, Singapore, 1995), p. 457.

${ }^{25}$ For a review, see R. Shanker, in Low-dimensional Quantum Field Theory for Condensed Matter Physicists (Ref. 24), p. 353.

${ }^{26}$ N. M. Bogoliubov and V. E. Korepin, Int. J. Mod. Phys. B 3, 427 (1989).

${ }^{27}$ K. B. Efetov and A. L. Larkin, Sov. Phys. JETP 41, 76 (1975); A. A. Abrikosov, J. Low Temp. Phys. 53, 359 (1983).

${ }^{28}$ A. G. Lebed, JETP Lett. 44, 114 (1986); N. Dupuis, G. Montambaux, and C. A. R. Sa de Melo, Phys. Rev. Lett. 70, 2613 (1993).

${ }^{29}$ M. Rasolt and Z. Tesanovic, Rev. Mod. Phys. 64, 709 (1992), and references therein.

${ }^{30}$ D. F. Agterberg, T. M. Rice, and M. Sigrist, Phys. Rev. Lett. 78, 3374 (1997); I. I. Mazin and D. J. Singh, ibid. 79, 733 (1997).

${ }^{31}$ For a review, see D. Vollhardt and P. Wölfle, The Superfluid Phases of Helium 3 (Taylor \& Francis, London, 1990).

${ }^{32}$ I. J. Lee et al., Phys. Rev. Lett. 78, 3555 (1997).

${ }^{33}$ Y. Hasegawa and H. Fukuyama, J. Phys. Soc. Jpn. 56, 877 (1987). 